\title{
COMPETENCY OR DUTY - METHODS OF SELECTION IN PRACTICE
}

My work deals with the connection between selection methods and competencies. It seeks an answer how company managers interpret the notion of competency, how important labour selection is according to those competencies. The choice among these methods is determined by conscious or rather subjective aspects, traditions and company culture. The findings are the partial results of a current, practical research. The subject is of a significant importance, as there are hardly any researches dealing with selection and its methods. However, its practical importance is beyond doubt, since the reason for the efficiency of organizations and the only resource of permanent competitive edge is the individual. This fact requires the use of selection methods that provide the satisfaction of employees beyond company efficiency.

\section{The importance of the topic}

During my work as a lecturer and counsellor, which I have been doing for several years now, my scope of interest has more and more turned to the study of labour force selection. I have indulged into this field so much that I have chosen it for my $\mathrm{PhD}$ research and MBA thesis. The scope of the research is selection among selection methods. I aim at finding its scientific or practical, subjective or objective reasons and expedience. I believe these methods form an inseparable unity with competencies. There are, however, further difficulties with this interpretation: there is no standard definition for competency in company practice - possibly not even in technical literature. There exist different interpretations for the types and classes of competency. Without standard notions, the strategic importance of HR management can hardly be maintained. My practical research has been going on now with four multinational firms. Three firms are in the Middle-West Transdanubian Region, two of them in FMCG sector, one is a supplier for car industry. The fourth one produces informatics appliances in the agglomeration of Budapest.

Recently, HR management has been more and more in the centre of interest for researchers dealing with organizations. The importance of this field has not only grown in the world of science but in practical life since its representatives appeared in the higher management of several organizations. The reason for this, according to the latest trends in management, is that the efficiency of organizations depends on the individual. The only and most durable source of competitive advantage is the individual and his/her ability to learn and improve. The individual is capable of improving the organization consciously and embedding values which promote creative, innovative thinking, continuous change and adaptation to the environment.
These days more and more organizations employ highly qualified people. By doing so, a definite demand emerges for the efficiency of selection from HR departments. Successful selection helps the firm and makes its work easier as well as it increases motivation and satisfaction of employees. Besides, it reduces the costs, having a positive effect on the firm's economy.

\section{Selection methods}

According to present literature, various methods are used in both intrinsic and extrinsic selection. In my study I consider the following methods:

- application forms

- CV

- tests

- interviews

- graphology

- test work

- references

- expert examination (e. g. health certificate)

- assessment centre (AC)

\section{Competency}

As a result of my research and Internet browsing, here I present some descriptions of competency to prove how ambiguously this notion is used in literature.

"Competency is the ability we need in an extremely wide range of cases. It is not only the use of learned skills in new situ-

\footnotetext{
* Gabor Fejes,

Budapest Tech Regional Education and Innovation Centre Szekesfehervar, Budai road 45. 8000 Hungary, E-mail: fejesg@strategia-taktika.hu
} 
ations but the complexity of inherent features, life experience, and other factors, intuitive mechanisms" [1].

"Competency is something by which we are able to fulfil tasks constructively in newer and newer situations in the world" [2].

"Competency resembles the features of a personality, skill, motivation or knowledge, which manifest themselves in behaviour. However, competency is above all, and includes all of them" [2].

"Competency consists of five factors:

- knowledge and ideas;

- skills and expertise;

- individual values;

- personal features;

- motivation"[2].

"Competencies are the personal features which make employees efficient and successful in a particular position or role"[2].

"Competency: skill and expertise"[3].

"Competency is a skill to fulfil a particular task or activity."

" Competency is the complexity of skills needed to do a job."

"Competency is the complexity of technical skills."

"Competency is a system of skills recognized, assessed and accepted by a particular firm." (The 4 quotations above come from the feedback from firm leaders.)

In what follows I use the notion of competency as Boyatzis defined it.

"Competency is the basic features of an individual which affect the efficiency and/or the result in compliance with the criteria." [4]

As for the types of competencies, I follow Vekerdy's typology [5]. Competency includes:

1. Skills and knowledge, which are important factors of what an individual is able to do if he/she wants. However, this knowledge does not necessarily mean that he/she does want to do it.

2. Skills and expertise which mean the possibility to realize the acquired knowledge in practice.

3. Social roles which form along individual values. The question "what an individual considers to be a value"

4. Self image, the image that the individual has about him/herself. Its important construent is reality and self-knowledge.

5. Individual features:

- Dimensions of the senses. Reactions to the stimuli coming from the environment. The questions "what we sense" and "how we react" are especially important.

- Cognitive skills. Features of memory, imagination and thinking.

- Psychosocial factor: self-estimation, self-confidence, independence etc.

6. Motivation: drive for action and behaviour. Wish and desire can also be found in this area.

7. Attitude: emotional, mental and behavioural approach to things through direct or indirect experience.

Competency always includes intension for effective action. Competency used in selection is usually competency related to work and behaviour, which are the following:
- Threshold competency needed for everyone to do a particular job, but it does not make a difference between the outstanding and the average,

- Performance competency means the factors that can make a difference between the outstanding and the average.

\section{Connection between selection and competency}

The most important aim of selection is to help decide who can and who cannot be a member of the organization. The person whose competency fits better to the job requirements and who is more likely to prove to be good within a particular period of time can be a member of the organization.

We can decide if a person fits or not if the comparison is made in the same dimension. I believe the most suitable dimension is competency. The selection system must be based on competency because job requirements as demands for competency must be comparable, commensurable with the competency inventory of the individual. Different areas can be compared, such as learned skills, experience, dealing with connections, problem solving, decision making, taking responsibility, motivation, leadership abilities, loadability etc. Every organization looks for individuals with the features that promote success for the organization. Selection is a key question because the organization cannot gain success without it.

Competency-based resource management and especially selection makes the opportunity for high efficiency. The introduction of the system has started but it is only in its enfancy. Traditional staff duties focused on different values, which generally decreased the individuals' self-knowledge, self-esteem and self-estimation. As opposed to this, it was a great change for Hungarian companies that they suddenly switched to be profit-centred. Value crisis of particular human features and competency does not help spread competency-based HR thinking. We must admit that the top management of Hungarian firms has hardly been interested in such problems until now. What is more, the strategic perspective has not been reflected in the selection function of HR management so far. Where efforts were made to solve the problem, they tried to do it as simply and fast as possible, slobbering or ignoring the moral and emotional process of the making values systematically.

In my opinion, its consequences are the following:

- The organization's demand for competency and the competency of employees must agree.

- The organization's demand for competency can only be defined clearly and exactly for a particular moment. A difference must be made between revealed and unrevealed demand for competency.

- Individual competency cannot be completely explored, therefore, a difference must be made between known and unknown demand for competency.

- Unrevealed and unknown demand for competency brings uncertainty into the HR system and the management of knowledge. 
- Inefficiency may result not only from inadequacy but from insufficient or lacking exploration of competency demands, so admittance criteria will not be reasonable enough.

- If an individual's known demand of competency falls short of performance, the missing competency may be compensated by planned skill improvement

- An employee can supply his/her own missing competency by self-education.

In a nutshell, we can state that accuracy, reliability, acceptability, clarity and unanimity are crucially important for a well functioning competency system. Since a longer time is needed to develop competencies, they must be ranked by firms for their future success If it is all done, they only need to find the suitable individuals to meet these competencies. The last step in the process of successful competency-based selection is to integrate the recruited employees-to-be into the organization. By doing so, we will be able to save them for the organization and to their skills entirely for our longterm success and the preservation of our competitive power.

\section{Practical experience}

The findings of my research are based on a written feedback given by HR managers and structured interviews made with them. The most important findings are the following:

Human resources have a strategic importance for every company, however, it is not so obvious in the daily practice. Sentences like "Get me a good employee for tomorrow" do not reinforce the faith put in the strategic importance of HR. It is interesting that managers believe they are infallible. It can also be noticed in the case of selection officers. They feel they are wise enough to select candidates on the base of their intuitions and experience. However, if they are not objective with the methods they cannot be objective with the results either. Most of them do not know all the methods and their introduction and use are the result of personal beliefs, company culture and traditions. During the process of selection, a key factor is how problem-oriented the managers are. Today, a good candidate should be employed not only in the case of vacancy but if he/she applies! If we accept the theory that every company needs key figures, then it is appropriate to employ a candidate with the proper competency as a potential key figure by creating a position for him/her.
Experts of HR selection rarely initiate changes in their field. They generally accept the established company practice as far as technologies and methods are concerned. The reason for this is very often inflexibility, subjectivism, and resistance against changes.

An important conclusion is the inadequate use of internal selection opportunities. The reason for this is the complete lack of employees' competency inventories. Therefore, internal selection can only bring success randomly. If there is an employee who is willing to apply. Apparently, it will fall through if the employee is rejected. $\mathrm{He} / \mathrm{she}$ will be a condemned person who is obviously to leave and will be dealt accordingly. I believe the other crucial problem is the inner communication channel. There are different practices for this, but it is mostly based on traditions. The opportunities of the inner company network could be used more efficiently but for this it is essential to know the expected and employees' competency. Most of the firms I examined have already defined the system of the expected competency, competency levels and classes. The direction of further development can only be to break down competency according to tasks (jobs), since besides the basic and key competencies - which are expected to be acquired by every employee - there exist functional competencies which are expected only from particular groups of employees.

As a conclusion, it can be stated that the necessity of competency-based selection has been recognized by experts. Companies have started to define and standardize the expected competencies and work out their competency model. Unfortunately, the interpretation of competency works on the level of the organization so far. No company has started to break down them down into the jobs and activities, which is the only way to proceed. Human resources are seen as a strategic factor everywhere, however, the functional units of companies do not deal with the activities of HR departments as a key factor but as a service. Subjective procedures are important beside the efforts for the enhancement of selection objectivity. The subjectivism of officers does not necessarily lead to the selection of the most efficient methods. The calculation of selection costs by HR controlling is only its infancy. Apart from raising the standards, the recognition of the importance of competency-based selection forms a firm basis of company efficiency, employees' motivation and satisfaction and the proper functioning of the company's communication process.

\section{References}

[1] UDVARDY, LAPOS, E.: Competence, Modularity, Paradigm Change in Practice (in Hungarian), www.galileo.ktk.pte.hu; <http://www.galileo.ktk.pte.hu/>, 23/03/2006

[2] www.odpartner.hu <http://www.odpartner.hu/>, 23/03/2006

[3] Dictionary of the Hungarian Language (in Hungarian), Akadémiai Kiadó, Budapest, 2003

[4] ELBERT, N. F., KAROLINY, M., FARKAS F., POÓR, J.: Manual of Staff / Human Resources Management (in Hungarian), KJKKERSZÖV Jogi és Üzleti Kiadó Kft., Budapest, 2002

[5] VEKERDY, I.: Human Resources Management I. (in Hungarian), Szent István Egyetem, Gödöllő, 2004 